\title{
An update on airborne contact dermatitis: 2001-2006
}

\author{
Raquel Santos ${ }^{1}$ and An Goossens ${ }^{2}$ \\ ${ }^{1}$ Department of Dermatology, Hospital de Curry Cabral, P-1069-166 Lisboa, Portugal \\ and ${ }^{2}$ Department of Dermatology, University Hospital K.U. Leuven, \\ B-3000 Leuven, Belgium
}

\begin{abstract}
Reports on airborne dermatoses are mainly published in the context of occupational settings. Hence, in recent years, dermatologists and also occupational physicians have become increasingly aware of the airborne source of contact dermatitis, resulting mainly from exposure to irritants or allergens. However, their occurrence is still underestimated, because reports often omit the term 'airborne' in relation to dust or volatile allergens. For the present update, we screened the journals 'Contact Dermatitis' (July 2000 to December 2006); 'Dermatitis', formerly named 'American Journal of Contact Dermatitis'; 'La Lettre du Gerda' (January 2000 to December 2006); and also included relevant articles from other journals published during the same period. This resulted in an updated list of airborne dermatitis causes.
\end{abstract}

Key words: airborne; allergic contact dermatitis; contact urticaria; irritant contact dermatitis; occupational; photo-allergic contact dermatitis. (c) Blackwell Munksgaard, 2007.

Accepted for publication 7 June 2007

Contact dermatitis is defined 'airborne' on the basis of: (i) the presence in the environment of dust, droplets, or volatile causative agents; (ii) the clinical symptoms; (iii) the history of the patient and the follow-up; and (iv) the results of epicutaneous tests.

The nature of airborne reactions can be various, among which irritant, allergic, photoallergic, phototoxic, and contact urticarial, being the most common; some agents may induce more than 1 type of reaction and sometimes 1 dermatitis may mask another 1, such as for example, in the case of rosacea and airborne dermatitis in a farmer (1).

Reports on airborne dermatoses are mainly published in the context of occupational settings (2-8). Hence, in recent years, dermatologists and also occupational physicians have become increasingly aware of the airborne source of contact dermatitis, resulting mainly from exposure to irritants or allergens. Airborne contacts are still greatly underestimated although, because reports often omit this term in relation to dust or volatile allergens ( 9 , 10). In some cases although, the responsible agents have been isolated by means of chemical analysis or direct microscopic study of the air or materials in the air (11). The different routes of exposition and relation to the clinical picture are sometimes misunderstood as well $(12,13)$.
The role of aeroallergens as a cause of allergic contact dermatitis or 'allergic contact dermatitislike' atopic dermatitis is controversial. Occasionally, inhalation of pollens, dusts, and animal hair causes either flare-up of atopic dermatitis, or an apparent superimposed contact dermatitis; in some instances, the airborne allergens may produce positive patch-test reactions (i.e. with dermatophagoides, 14).

For the present update, we screened the Contact Dermatitis (July 2000 to December 2006); the Dermatitis, formerly named American Journal of Contact Dermatitis, and La Lettre du Gerda (January 2000 to December 2006); and also included relevant articles from other journals published during the same period. Table 1 gives the lists (that do not pretend to be exhaustive) with the airborne causes of dermatoses that we could retrieve. For previous reviews on this subject, we refer to Huygens and Goossens (2) and Lachapelle (10), the latter regarding irritant contact dermatitis.

Most of the allergens identified were in an occupational setting, if not the references are indicated with asterisk (Table 1). As in other recent reviews $(2,15,16)$ plants represent a very important cause, particularly the Asteraceae (Compositae) family, for which recent advances in investigation of the clinical 
Table 1. Causes of airborne dermatosis ${ }^{\mathrm{a}}$

References

Allergic contact dermatitis

Plants, natural resins, and wood allergens

Ambrosia deltoidea (triangle-leaf bursage)

Cashew nut (family Anacardiacea)

$(22 *)$

Chamomilla recutita (German chamomile)

Champignon

$(24 *)$

Chrysanthemum

$(25)$

Colophonium

(26)

Compositae

Evernia prunastri (oak moss)

$(27,28,29)$

Frullania

$(30 *, 31 *)$

(32)

Gaillardia

(34)

Garlic

Humulus lupulus

Iris (lis)

(36)

(37)

Latex

Laurus nobilis

Machaerium schleroxylon (Santos rosewood)

(38)

$(39 *)$

Parthenium hysterophorus

Picae abies (spruce)

Pinus sylvestris (Finish pine)

Primula obconica

Propolis

Pterocarpus soyauxii Taub (Padauk wood)

$(40,41)$

$\left(42^{*}, 43^{*}, 44^{*}, 45^{*}, 46^{*}\right)$

(47)

(47)

$(48,49)$

$(50,51 *, 52)$

(53)

Rosmarinus officinalis

Swartizia madagascariensis

$(54,55)$

(56)

Thymus vulgaris (Thyme)

Triplochiton scleroxylon (Obeche)

Tropical woods: Dalbergia retusa (Cocobolo wood)

(57)

(58)

Verbascum densiflorum (gordolobo)

$(40,59)$

Plastics, rubbers, and glues

Acrylates

Benzoyl peroxide

Epoxy acrylates

Epoxy resin (and amines)

Formaldehyde and formaldehyde resins

Isocyanates

Metals

Cobalt

$(61-64)$

$(65,66)$

(67)

$(68,69-82)$

$(83,84)$

$\left(85,86^{*}, 87,88,89\right)$

Mercury

(90)

Nickel

$(91,92)$

Silver

$(93,94)$

Industrial and pharmaceutical chemicals

2-Aminothiophenol

p-Aminophenol

(96)

Azathioprine

Azithromycin

n-Alkyl dimethylbenzylammonium chloride

$(97 *)$

$(98,99)$

$N, N$-bis[2-bromo-ethyl] aniline

$(100)$

Budesonide

(101)

(102)

2-Butin-1,4-diol

(103)

Ceftiofur

(104)

Cyanamide and dibenzyl phosphite

(105)

Cinnamal (cinnamic aldehyde

(106)

Chloroacetamide

Diacetylmorphine

Diethylene glycol monobutyl ether

$(107 *)$

$(108,109)$

Ethacridine lactate

(110)

Formaldehyde and releasers (quaternium -15)

(111)

Glutaraldehyde

(112)

HBTU (o-(benzotriazol-1-y1)- $N, N, N^{\prime}, N^{\prime}$,-tetramethyluronium)

(113)

3-Iodo-2-propynyl-butylcarbamate

(114)

Isothiazolinones

(115)

Lansoprazole

$(116-118,119 *)($ Fig. 1)

Meropenem

$(120,121)$

Mesna

(122)

(123)

Methotrexate 
Table 1. Continued

\begin{tabular}{|c|c|}
\hline & References \\
\hline Methyl chloroform & $(125)$ \\
\hline ( $N$-methyl- $N$-nitroso-p-toluenesulfonamide) Diazald & $(126)$ \\
\hline Oxycodone & $(127)$ \\
\hline Paraphenylenediamine & $(128)$ \\
\hline Pristinamycin & $(129)$ \\
\hline Quinazoline oxide & $(130)$ \\
\hline Ranitidine & $(131)$ \\
\hline Simvastatin & $(132)$ \\
\hline Tetrazepam & $(133,134,135)$ \\
\hline Succinimidyl carbonates & $(136)$ \\
\hline \multicolumn{2}{|l|}{ Pesticides and animal feed additives } \\
\hline Acephate & $\left(137^{*}\right)$ \\
\hline 1,3-Dichloropropene & $(138)$ \\
\hline Olaquindox & $(139)$ \\
\hline Organophosphorus pesticides & $\left(140^{*}\right)$ \\
\hline Phoxim & $\left(141^{*}\right)$ \\
\hline Pigs' feed & $(142)$ \\
\hline Propineb & $(143)$ \\
\hline \multicolumn{2}{|l|}{ Miscellaneous } \\
\hline Agricultural dusts (storage mites, flax allergens, Pantoea agglomerans, and moulds) & $(5)$ \\
\hline Cigarettes & $\left(144^{*}\right)$ \\
\hline Disperse dyes & $(145)$ \\
\hline FAZ-exhaust Kodak (polyethylene terephtalate, polyvinil butyral, and silver) & $(146)$ \\
\hline \multicolumn{2}{|l|}{ Irritant contact dermatitis } \\
\hline Dermatitis in a particleboard manufacturing facility & $(147)$ \\
\hline Phosphates in a fertilizer factory & $(148)$ \\
\hline Synthetic fibres from an air-conditioning filter & $(149)$ \\
\hline Chlorothalonil & $(150)$ \\
\hline \multicolumn{2}{|l|}{ Photoallergic reactions } \\
\hline Carprofen & $(151)$ \\
\hline Chlorpromazine & $(152)$ \\
\hline \multicolumn{2}{|l|}{ Contact urticaria } \\
\hline Amoxicillin & $(153)$ \\
\hline Curcumin & $(154)$ \\
\hline Epoxy resin & $(155)$ \\
\hline HATU and HBTU & $(156,157)$ \\
\hline Hyacinth & $(158)$ \\
\hline Pine processionary caterpillar (Thaumetopoea pityocampa) & $(159-164)$ \\
\hline Spathiphyllum wall_isii flower & $(165,166)$ \\
\hline \multicolumn{2}{|l|}{ Weeping fig (Ficus benjamina) } \\
\hline \multicolumn{2}{|l|}{ Yucca (Yucca aloifolia) } \\
\hline \multicolumn{2}{|l|}{ Contact urticaria syndrome } \\
\hline Anisakis simplex & $(167,168)$ \\
\hline Compositae & (169) \\
\hline Diphenylmethane- $4,4^{\prime}$-diisocyanatae & $(170)$ \\
\hline Fern & $(171)$ \\
\hline Goat dander & $(172)$ \\
\hline Protease & $(173)$ \\
\hline Lupine flour & $(174)$ \\
\hline Triphenyl phosphite & $(175)$ \\
\hline \multicolumn{2}{|l|}{ Protein contact dermatitis } \\
\hline Flour & $(176)$ \\
\hline Sapele wood & $(177)$ \\
\hline \multicolumn{2}{|l|}{ Erythema-multiphorme-like eruption } \\
\hline 1,2-Ethanedithiol & $(178)$ \\
\hline Japanese lacquer tree (Rhus verniciflua) & $(179)$ \\
\hline Weeds & $(180)$ \\
\hline
\end{tabular}

${ }^{\mathrm{a}}$ References with asterisk $(*)$ represent non-occupational setting. HATU, $N$-[(dimethylamine)-1 $H$-1,2,3-triazolo[4,5,-b]pyridine-1-ylmythelene]- $N$-methyl-methanaminium hexafluorophosphate $N$-oxide; HBTU, $N$-[(1H-benzotriazol-1-yl)(dimethylamino)methylene]$N$-methylmethanaminium heafluorophosphat $N$-oxide) and 9-f.

signs, diagnosis, and therapy have been published $\left(17,18^{*}, 19 *, 20,21\right)$. Although mono- and sesquiterpene-lactones, contained in Asteraceae, are by far the most frequently described airborne allergens in weed dermatitis, many other plant and wood aller- gens remain unidentified. The principal sensitizers although include phenols (Anacardiaceae), quinones (Tectona, rosewood), and terpenes (Frullania, Pinus). Although airborne irritants are more difficult to demonstrate, they are certainly more common 


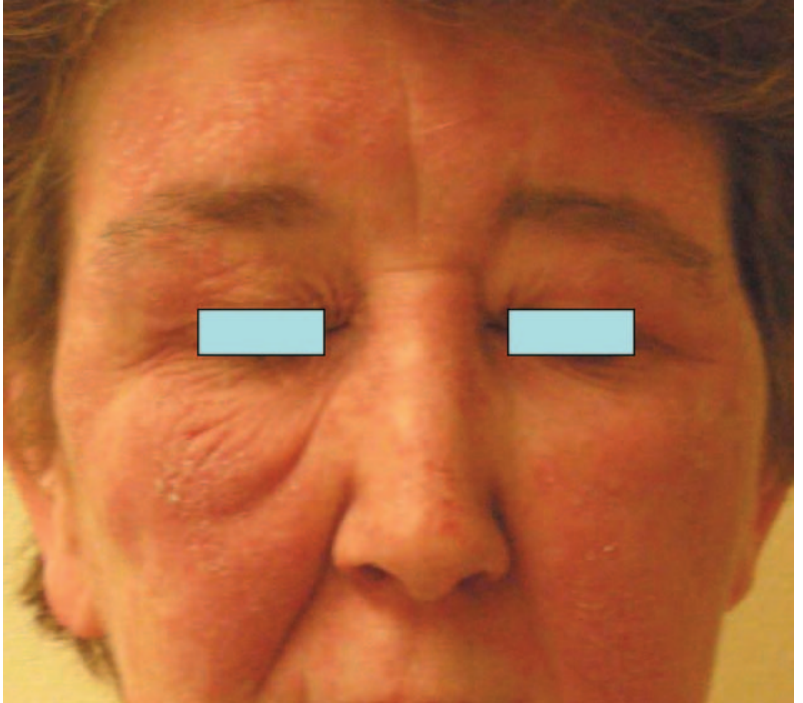

Fig. 1. Severe allergic contact dermatitis from airborne exposure to methyl- and methylchloro-isothiazolinone in freshly-painted walls.

than allergic reactions (10). They include fibrous materials (such as glass fibres, rock wool, and grain dust), which give rise to mechanical dermatitis by friction, inducing both hyperkeratosis and acute dermatitis; wood and cement dust, which cause irritant reaction with lichenified dermatitis at the contact sites.

\section{Conclusion}

This survey provides an updated list of airborne causes of dermatoses, most often occupationinduced. Irritant and allergic dermatitis are the most common, but some causes of photoallergic reactions, the contact urticaria (syndrome), protein contact dermatitis, and erythema-multiformelike eruptions have also been retrieved. The causes are multiple: plants, natural resins, and wood allergens; plastics, rubbers, and glues; metals; industrial and pharmaceutical chemicals; pesticides and animal feed additives, enzymes, and animal dander.

\section{References}

1. Spiewak R, Dutkiewicz J A. Farmer's occupational airborne contact dermatitis masqueraded by coexisting rosacea: delayed diagnosis and legal acknowledgement. Ann Agric Environ Med 2004: 11: 329-333.

2. Huygens S, Goossens A. An update on airborne contact dermatitis. Contact Dermatitis 2001: 44: 1-6.

3. Estlander T, Jolanki R, Alanko K, Kanerva L. Occupational allergic contact dermatitis caused by wood dusts. Contact Dermatitis 2001: 44: 213-217.

4. Gielen K, Goossens A. Occupational allergic contact dermatitis from drugs in healthcare workers. Contact Dermatitis 2001: 45: 273-279.
5. Li L, Sujan S A, Wang J. Detection of occupational allergic contact dermatitis by patch testing. Contact Dermatitis 2003: 49: 189-193.

6. Schmutz J-L, Barbaud A, Trechot P. Allergies de contact professionnelles et substances à visée thérapeutique. Ann Dermatol Venereol 2003: 130: 583.

7. Leggat P A, Smith D R. Dermatitis and aircrew. Contact Dermatitis 2006: 54: 1-4.

8. Quirce S, Escudero C, Swanson M C, MartínezAramayona M J, Fernández-Nieto M, Sastre J. Latex aeroallergen concentrations in ambulances. $J$ Allergy Clin Immunol 2004: 114: 978.

9. Chew A L, Maibach H I. Occupational issues of irritant contact dermatitis. Int Arch Occup Environ Health 2003: 76: 339-346.

10. Lachapelle J M. Airborne irritant dermatitis. In: Irritant Dermatitis, Chew A L, Maibach H I (eds). Berlin, Springer, 2006: 71-78

11. Reinhard E, Waeber R, Niederer M, Maurer T, Maly P, Scherer S. Preservation of products with $\mathrm{MCI} / \mathrm{MI}$ in Switzerland. Contact Dermatitis 2001: 45: 257-264.

12. Elberling J, Linneberg A, Mosbech H, Dirksen A, Frølund L, Madsen F, Nielsen N H, Johansen J D. A link between skin and airways regarding sensitivity to fragrance products? Br J Dermatol 2004: 151: 1197-1203.

13. Elberling J, Linneberg A, Mosbech H et al. Airborne chemicals cause respiratory symptoms in individuals with contact allergy. Contact Dermatitis 2005: 52: 65-72.

14. Davis M D, Richardson D M, Ahmed D D. Rate of patch test reactions to a Dermatophagoides mix currently on the market: a mite too sensitive? Am J Contact Dermat 2002: 13: 71-73.

15. Komericki P, Abere R W, Kranke B. An 8-year experience in air-borne contact dermatitis. Wien Klin Wochenschr 2004: 116: 322-325.

16. Proietti L, Bonanno G, Vasta N, Augeri M, Marconi A, Stivala F. Airborne Contact Dermatitis. Clin Ter 2006: 157: 531-539.

17. Sharma V K. Patch testing with the European standard series and Compositae extracts in patients with airborne contact dermatitis. Contact Dermatitis 2001: 44: 41-42.

18. Handa S, Sahoo B, Sharma V K. Oral hyposensitization in patients with contact dermatitis from Parthenium hysterophorus. Contact Dermatitis 2001: 44: 279-282.

19. Paulsen E, Christensen L P, Andersen K E. Do monoterpenes released from feverfew (Tanacetum parthenium) plants cause airborne Compositae dermatitis? Contact Dermatitis 2002: 47: 14-18.

20. Sharma V K, Sethuraman G, Tejasvi T. Comparison of patch test contact sensitivity to acetone and aqueous extracts of Parthenium hysterophorus in patients with airborne contact dermatitis. Contact Dermatitis 2004: 50: 230-232.

21. Sharma V K, Sethuraman G, Bhat R. Evolution of clinical pattern of parthenium dermatitis: a study of 74 cases. Contact Dermatitis 2005: 53: 84-88.

22. Schumacher M J, Silvis N G. Airborne contact dermatitis from Ambrosia deltoidea (triangle-leaf bursage). Contact Dermatitis 2003: 48: 212-216.

23. Criado R F, Criado P R, Malaman F, Ensina L F, Vasconcellos C, Aun W T, Mello J F, Pires M C. Nonoccupational allergic contact dermatitis to cashew nut simulating photosensitivity eczema. Am J Contact Dermat 2002: 13: 85-86.

24. Rycroft R J G. Recurrent facial dermatitis from chamomile tea. Contact Dermatitis 2003: 48: 229.

25. Simeoni S, Puccetti A, Peterlana D, Tinazzi E, Lunardi C Occupational allergic contact dermatitis from champignon and Polish mushroom. Contact Dermatitis 2004: 51: 156-157.

26. Groenewoud G C M, Jong N W, Burdorf A, de Groot H, Gerth van Wÿk R. Prevalence of occupational allergy to 
Chrysanthemum pollen in greenhouses in the Netherlands. Allergy 2002: 57: 835-840.

27. Alvarez-Cuesta C C, López F V, Aguado C R, López M A G, Oliva N P. Allergic contact dermatitis from colophonium in the sawdust of Asturian cider-bars. Contact Dermatitis 2001: 45: 57.

28. Agarwal S, Gawkrodger D J. Occupational allergic contact dermatitis to silver and colophonium in a jeweller. $\mathrm{Am} \mathrm{J}$ Contact Dermat 2002: 13: 74.

29. Corazza M, Borghi A, Virgili A. A medicolegal controversy due to a hidden allergen in cutting oils. Contact Dermatitis 2004: 50: 254-255.

30. Fortina A B, Romano I, Peserico A. Contact sensitization to Compositae mix in children. $J$ Am Acad Dermatol 2005: 53: 877-880.

31. Killoran C, Pedvig-Leftick A, Williams R. A case of compositae airborne dermatitis exarcebated by moisturizer containing feverfew. Dermatitis 2006: 17: 104.

32. Owen C M, August P J, Beck M H. Contact allergy to oak moss resin in a soluble oil. Contact Dermatitis 2000: 43: 112.

33. Julian C G, Bowers P W, Paton J A. Frullania dermatitis. Contact Dermatitis 2000: 43: 119-120.

34. Foin N G. Sensabilisation de contact a une Asteracée: La Gaillarde. La Lettre du Gerda 2005: 19: 25-28.

35. Bassioukas K, Orton D, Cerio R. Occupational airborne allergic contact dermatitis from garlic with concurrent Type I allergy. Contact Dermatitis 2004: 50: 39-41.

36. Spiewak R, Dutkiewicz J. Occupational airborne and hand dermatitis to hop (Humulus lupulus) with non-occupational relapses. Ann Agric Environ Med 2002: 9: 249-252.

37. Dejobert Y. Allergie à l'iris. La lettre du Gerda 2001: 18:38-39.

38. Nettis E, Colanardi M C, Ferrannini A, Tursi A. Airborne allergic contact dermatitis. Allergy 2001: 56: 583-584.

39. Özden M G, Oztas P, Öztas M O, Onder M. Allergic contact dermatitis from Laurus nobilis (laurel) oil. Contact Dermatitis 2001: 45: 178.

40. Correale C E, Marks J G Jr. Contact dermatitis in a woodworker. Am J Contact Dermat 2002: 13: 42-44.

41. Wong Y W, Powell S M. Contact dermatitis in a bagpipes maker. Contact Dermatitis 2003: 49: 310.

42. Sahoo B, Handa S, Kumar B. Eczema herpeticum in parthenium dermatitis. Contact Dermatitis 2001: 44: 106-107.

43. Verma K K, Sirka C S, Ramam M, Sharma V K. Parthenium dermatitis presenting as photosensitive lichenoid eruption. A new clinical variant. Contact Dermatitis 2002: 46: $286-289$.

44. Mahajan V K, Sharma N L, Sharma R C. Parthenium dermatitis: is it a systemic contact dermatitis or an airborne contact dermatitis? Contact Dermatitis 2004: 51: 231-234.

45. Prabhakar A C, Dogra S, Handa S. Eczema herpeticum complicting parthenium dermatitis. Am J Contact Dermat 2005: 16: 78-79.

46. Singh M N, Beck M H. Parthenium contact sensitivity travels to the U.K. Br J Dermatol 2006: 155: 847-848.

47. Majamaa H, Viljanen P. Occupational facial allergic contact dermatitis caused by Finnish pine and spruce wood dusts. Contact Dermatitis 2004: 51: 157-158.

48. Aplin C G, Lovell C R. Contact dermatitis due to hardy primula species and their cultivars. Contact Dermatitis 2001: 44: 23-29.

49. de Miguel J, Quirce S, Escudero C, de las Heras M, Cuesta J, Sastre J. Occupational airborne allergic contact dermatitis from Primula obcónica. Allergy 2002: 57: 305.

50. Lieberman H D, Fogelman J P, Ramsay D L, Cohen D E. Allergic contact dermatitis to propolis in a violin maker. J Am Acad Dermatol 2002: 46: S30-S31.

51. Tobin A, Kirby B. Airborne contact dermatitis induced by a neighbour's beehives. Contact Dermatitis 2003: 49: 214-215.

52. Fernandez S G, Alemán E A, Figueroa B E G, Estibaliz G F, Rivera J M O, Purroy A I T. Direct and airborne contact dermatitis from propolis in beekeepers. Contact Dermatitis 2004: 50: 320-321.

53. Kiec-Swierczynska M, Krecisz B, Swierczynska-Machura D, Palczynski C. Occupational allergic contact dermatits caused by padauk wood (Pterocarpus soyauxii Taub.) Contact Dermatitis 2004: 50: 384-385.

54. Serra E, Vila A, Peramiquel L, Dalmau J, Granel C, Alomar A. Allergic contact dermatitis due to rosemary. Contact Dermatitis 2005: 53: 179-180.

55. González-Mahave I, Lobesa T, Del Pozo M D, Blasco A, Venturini M. Rosemary contact dermatitis and crossreactivity with other labiate plants. Contact Dermatitis 2006: 54: 210-212.

56. Almeida Pereira M, Barros A M, Cunha A P, Lisboa C, Azevedo F. Airborne allergic contact dermatitis from Swartizia madagascariensis. Contact Dermatitis 2006: 55: 33.

57. Spiewak R, Skorska C, Dutkiewicz J. Occupational airborne contact dermatitis caused by thyme dust. Contact Dermatitis 2001: 44: 235-239.

58. Kanerva L, Leino T, Estlander T. Occupational allergic contact dermatitis in carpenters. Contact Dermatitis 2001: 45: 61-62.

59. Guanche A D, Prawer S. Generalized eczematous contact dermatitis from cocobolo wood. Am J Contact Dermat 2003: 14: 90

60. Castro A I, Carmona J B, Gonzales F G, Nestar O B. Occupational airborne dermatitis from gordolobo (Verbascum densiflorum). Contact Dermatitis 2006: 55: 301.

61. Bong J L, English J S C. Allergic contact dermatitis from airborne exposure to acrylates. Contact Dermatitis 2000: 43: 242.

62. Geukens S, Goossens A. Occupational contact allergy to (meth)acrylates. Contact Dermatitis 2001: 44: 153-159.

63. Moffitt D L, Sansom J E. Occupational allergic contact dermatitis from tetrahydrofurfuryl acrylate in a medicaldevice adhesive. Contact Dermatitis 2001: 45: 54-55.

64. Hickey J R, Sansom J E. Allergic contact dermatitis following airborne exposure to methacrylates used in the manufacture of artificial skin. Contact Dermatitis 2003: 49: 221.

65. Forschner K, Zuberbier T, Worm M. Benzoyl peroxide as a cause of airborne contact dermatitis in an orthopaedic technician. Contact Dermatitis 2002: 47: 241.

66. Tsovilis E, Crépy M, Jonathan A, Ameille J. Occupational contact dermatitis due to a marbler's exposure to benzoyl peroxide. Contact Dermatitis 2005: 52: 117-118.

67. Kanerva L, Estlander T, Jolanki R, Alanko K. Occupational allergic contact dermatitis from 2,2-bis(4-(2-hydroxy-3-acryloxypropoxy)phenyl)-propane (epoxy diacrylate) in ultravioletcured inks. Contact Dermatitis 2000: 43: 56-59.

68. Sasseville D, Moreau L, Brassard J, Leclerc G. Allergic contact dermatitis to epoxy resin in microscopy immersion oil: Cases from Canada. Am J Contact Dermat 2000: 11: 99-103.

69. Ahmed I, Ilchyshyn A. Immersion oil allergy with no reaction to epoxi resin in the standard series. Contact Dermatitis 2000: 43: 125-126.

70. el-Azhary R A, Yiannias J A. Allergic contact dermatitis to epoxy resin in immersion oil for light microscopy. $J \mathrm{Am}$ Acad Dermatol 2002: 47: 954-955.

71. Kanerva L, Estlander T, Keskinen H, Jolanki R. Occupational allergic airbone contact dermatitis and delayed bronchial asthma from epoxy resin revealed by bronchial provocation test. Eur J Dermatol 2000: 10: 475-477.

72. Kanerva L, Jolanki R, Estlander T, Henriks-Ecckerman M, Tuomi M, Tarvainen K. Airborne occupational allergic contact dermatitis from triglycidyl-p-aminophenol and tetraglycidyl-4,4'-methylene dianiline in preimpregnated epoxy products in the aircraft industry. Dermatology 2000: 201: 29-33.

73. Jappe U, Geier J, Hausen B M. Contact vitiligo following a strong patch test reaction to triglycidyl-p-aminophenol in 
an aircraft industry worker: case report and review of the literature. Contact Dermatitis 2005: 53: 89-92.

74. Ducombs G. Dermites de contact provoquées par les fibres de carbone et résins époxi. La Lettre du Gerda 2000: 17: 14.

75. Acciai M C, Sertoli A, Gola M, Vanni E, Calabretta V M, Carnevalli C. Dermatite allergica da contatto professionale da fibra di carbonio trattate con resine epossidiche. Annali italiani di dermatologia allergologica 2006: 60: 62-64.

76. Koch P. Occupational allergic contact dermatitis from epoxy resin systems and possibly acetone in a shoemaker. Contact Dermatitis 2002: 46: 362-363.

77. Machado S, Silva E, Sanches M, Massa A. Occupational airborne contact dermatitis. Am J Contact Dermat 2003: 14: 31-32.

78. Katugampola R P, Mills C M, Stone N M. Occupational allergic contact dermatitis to epoxy resin in a gravestone repairer. Contact Dermatitis 2006: 55: 194.

79. Yokota K, Michitsuji H. Occupational allergic contact dermatitis from 1,6-bis (2,3-epoxypropoxy) naphthalene in a one-component epoxy coating. Contact Dermatitis 2004: 51: 154-155.

80. Kirkup M E, Murphy J, Beck M H, Sansom J E. Occupational contact sensitization to 1,2-diaminocyclohexane. Contact Dermatitis 2001: 45: 121-122.

81. Geier J, Oestmann E, Lessmann H, Fuchs T. Contact allergy to terephthalic acid diglycidylester in a powder coating. Contact Dermatitis 2001: 44: 35-36.

82. Guin J D. Airborne contact dermatitis from a polymercaptan hardener in a finished epoxy resin. Contact Dermatitis 2005: 52: 45.

83. Ezughah F I, Murdoch S R, Finch T M. Occupational airborne allergic contact dermatitis from medium-density fibreboard containing phenol-formaldehyde resin-2 (PFR-2). Contact Dermatitis 2001: 45: 242.

84. Bell H K, King C M. Allergic contact dermatitis from ureaformaldehyde resin in medium-density fibreboard (MDF). Contact Dermatitis 2002: 46: 247.

85. Goossens A, Detienne T, Bruze M. Occupational allergic contact dermatitis caused by isocyanates. Contact Dermatitis 2002: 47: 304-308.

86. Morgan C J, Haworth A E. Allergic contact dermatitis from 1,6-hexamethylene diisocyanate in a domestic setting. Contact Dermatitis 2003: 48: 224.

87. Millitello G, Sasseville D, Ditrec C, Brod B A. Allergic contact dermatitis from isocyanates among sculptors. Dermatitis 2004: 15: 150-153.

88. White J M L, White I R. Allergic contact dermatitis from dicyclohexylmethane-4,4'-diisocyanate: two cases. $\mathrm{Br} J$ Dermatol 2005: 153: 58-59.

89. Hannu T, Estlander T, Jolanki R. Allergic contact dermatitis due to MDI and MDA from accidental occupational exposure. Contact Dermatitis 2005: 52: 108-109.

90. Dickel H, Radulescu M, Weyher I, Diepgen $T$ L. Occupationally-induced "isolated cobalt sensitization". Contact Dermatitis 2001: 45: 246-247.

91. Bartolome B, Cordoba S, Sanchez-Perez J, FernandezHerrera J, Garcia-Diez A. Baboon syndrome of unusual origin. Contact Dermatitis 2000: 43: 113.

92. Suzuki K, Matsunaga K, Umemura Y, Ueda H, Sasaki K. 2 cases of occupational dermatitis due to mercury vapor from a broken sphygmomanometer. Contact Dermatitis 2000: 43: 175-177.

93. Candura S M, Locatelli C, Butera R, Gatti A, Fasola D, Manzo L. Widespread nickel dermatitis from inhalation. Contact Dermatitis 2001: 45: 174-175.

94. Sertoli A, D’Aglino S, Parrini L, Carnevalli C, Vanni E. A non common cause of occupational sensitivity to nickel. Annali italiani di Dermatologia alergollogica 2005: 59: 125.

95. Bonamonte D, Carino M, Mundo L, Foti C. Occupational airborne allergic contact dermatitis from 2-aminothiophenol. Eur J Dermatol 2002: 12: 592-593.
96. Walker S L, Ead R D, Shackleton D B, Beck M H. Two cases of occupational allergic contact dermatitis to p-aminophenol in pharmaceutical workers manufacturing paracetamol. Contact Dermatitis 2005: 52: 290-291.

97. Lauerma A I, Koivuluhta M, Alenius H. Recalcitrant allergic contact dermatitis from azathioprine tablets. Contact Dermatitis 2001: 44: 129.

98. Milković-Kraus S, Kanceljak-Macan B. Occupational airborne allergic contact dermatitis from azithromycin. Contact Dermatitis 2001: 45: 184

99. Mimesh S, Pratt M. Occupational airborne allergic contact dermatitis from azithromycin. Contact Dermatitis 2004: 51: 151 .

100. Mauleón C, Mauleón P, Chavarría E, La Cueva P, Suárez R, Lázaro P. Airborne contact dermatitis from n-alkyl dimethylbenzylammonium chloride and n-alkyl dimethylethylbenzylammonium chloride in a detergent. Contact Dermatitis 2006: 55: 311-312.

101. Verlinden V, Goossens A. Airborne occupational allergic contact dermatitis from N,N-bis[2-bromo-ethyl] aniline and N,N-bis [2-[(methylsulfonyl)-oxy ]ethyl]aniline in a chemistry student. Contact Dermatitis 2003: 49: 169.

102. Pontén A. Airborne occupational contact dermatitis caused by extremely low concentrations of budesonide. Contact Dermatitis 2006: 55: 121-124.

103. Blaschke V, Reinecke S, Fuchs T. Allergic contact dermatitis from 2-butin-1,4-diol. Allergy 2001: 56: 264-265.

104. Antico A, Marcotulli C. Occupational contact allergy to ceftiofur. A case of extensive dermatitis for occupational contact allergy to ceftiofur (without cross-reactivity to other cephalosporins) is reported. Allergy 2003: 58: 957-958.

105. Foti C, Bonamonte D, Carino M, Mastrandrea V, Angelini G. Occupational airborne contact allergy to cyanamide and dibenzyl phosphite. Contact Dermatitis 2003: 48: 272-273.

106. Decapite T J, Anderson B E. Allergic contact dermatitis from cinnamic aldehyde found in an industrial odourmasking agent. Contact Dermatitis 2004: 51: 312-313.

107. Bogenrieder T, Landthaler M, Stolz W. Airborne contact dermatitis due to chloroacetamide in wall paint. Contact Dermatitis 2001: 45: 55.

108. Coenraads P J, Hogen Esch A J, Prevoo R L M A. Occupational contact dermatitis from diacetylmorphine (heroin). Contact Dermatitis 2001: 45: 114.

109. Hogen Esch A J, van der Heide S, van den Brink W, van Ree J M, Bruynzeel D P, Coenraads P J. Contact allergy and respiratory/mucosal complaints from heroin (diacetylmorphine). Contact Dermatitis 2006: 54: 42-49.

110. Schliemann-Willers S, Bavar A, Elsner P. Occupational contact dermatitis from diethylene glycol monobutyl ether in a podiatrist. Contact Dermatitis 2000: 43: 225.

111. Rudzki E, Rebandel P. Airborne contact dermatitis due to ethacridine lactate in a veterinary surgeon. Contact Dermatitis 2001: 45: 234

112. Zina A M, Fanan E, Bundino S. Allergic contact dermatitis from formaldehyde and quaternium-15 in a photocopier toner. Contact Dermatitis 2000: 43: 241-242.

113. Vyas A, Pickering C A, Oldham L A, Francis H C, Fletcher A M, Merrett T, Niven R M. Survey of symptoms, respiratory function, and immunology and their relation to glutaraldehyde and other occupational exposures among endoscopy nursing staff. Occup Environ Med 2000: 57: $752-759$.

114. Bousquet P-J, Guillot B, Guilhou J-J, Raison-Peyron N. Occupational airborne allergic contact dermatitis due to HBTU. Contact Dermatitis 2005: 52: 53-54.

115. Jensen C D, Thormann J, Andersen K E. Airborne allergic contact dermatitis from 3-iodo-2-propynyl-butylcarbamate at a paint factory. Contact Dermatitis 2003: 48: 155-157.

116. Kristensen D, Hein H O, Weismann K. Airborne contact allergy provoked by kathon in water-based plastic paint. Ugeskr Laeger 2002: 164: 2411-2413. 
117. Young H S, Ferguson J E F, Beck M H. Contact dermatitis from 2-n-octyl-4-isothiazolin-3-one in a $\mathrm{PhD}$ student. Contact Dermatitis 2004: 50: 47-48.

118. Hardcastle N J, Gawkrodger D J. Occupational contact dermatitis to 1,2-benzisothiazolin-3-one and 5-chloro-2methylisothiazolin-3-one/2-methyl-isothiazolin-3-one in paint manufacturers. Contact Dermatitis 2005: 53: 115-116.

119. Jensen J-M, Harde V, Brasch J. Airborne contact dermatitis to methylchloroisothiazolinone/methylisothiazolinone in a boy. Contact Dermatitis 2006: 55: 311.

120. Vilaplana J, Romaguera C. Allergic contact dermatitis due to lansoprazole, a proton pump inhibitor. Contact Dermatitis 2001: 44: 39-40.

121. Schena D, Germi L, Barba A, Costa G. Epidemic occupational allergic and irritant airborne contact dermatitis in pharmaceutical manufacture producing lansoprazole. Contact Dermatitis 2002: 46: 31.

122. Yesudian P D, King C M. Occupational allergic contact dermatitis from meropenem. Contact Dermatitis 2001: 45: 53.

123. Kiec-Swierczynska M, Krecisz B. Occupational airborne allergic contact dermatitis from mesna. Contact Dermatitis 2003: 48: 171 .

124. Dastychová E. Allergic contact dermatitis in methotrexate manufacture. Contact Dermatitis 2003: 48: 226.

125. Mallon J, Chu M T, Maibach H I. Occupational allergic contact dermatitis from methyl chloroform (1,1,1trichloroethane)? Contact Dermatitis 2001: 45: 107.

126. Young H S, Wilson N J E, Winter J, Beck M H. Contact dermatitis from Diazald. Contact Dermatitis 2004: 50: 315-316.

127. MacFarlane C S, Charman C, Fertig A, English J S C. An outbreak of contact dermatitis in an opiate manufacturing plant. Br J Dermatol 2003: 149: 95.

128. Dyche J A, Goldsmith P C. Case report of occupational sensitization to p-phenylenediamine in a microbiology laboratory. Br J Dermatol 2003: 149: 96.

129. Blancas-Espinosa R, Conde-Salazar L, Pérez-Hortet C. Occupational airborne contact dermatitis from pristinamycin. Contact Dermatitis 2006: 54: 63-65.

130. Rudzki E, Rebandel P. Contact sensitivity to quinazoline oxide. Contact Dermatitis 2001: 44: 49-50.

131. Ryan P J J, Rycroft R J G, Aston I R. Allergic contact dermatitis from occupational exposure to ranitidine hydrochloride. Contact Dermatitis 2003: 48: 67-68.

132. Peramiquel L, Serra E, Dalmau J, Vila A T, Mascaró J M, Alomar A. Occupational contact dermatitis from simvastatin. Contact Dermatitis 2005: 52: 286-287.

133. Choquet-Kastylevsky G, Testud F, Chalmet P, LecuyerKudela S, Descotes J. Occupational contact allergy to tetrazepam. Contact Dermatitis 2001: 44: 372.

134. Lepp U, Zabel P, Greinert U. Occupational airborne contact allergy to tetrazepam. Contact Dermatitis 2003: 49: 260-261.

135. Ferran M, Giménez-Arnau A, Luque S, Berenguer N, Iglesias M, Pujol R M. Occupational airborne contact dermatitis from sporadic exposure to tetrazepam during machine maintenance. Contact Dermatitis 2005: 52: 173-174.

136. Fowler J F Jr, Edge J C. Occupational airborne allergic contact dermatitis from succinimidyl carbonates. Contact Dermatitis 2001: 45: 38

137. Nakamura M, Miyachi Y. Airborne contact dermatitis caused by the pesticide acephate. Contact Dermatitis 2002: 47: 121-122.

138. Corazza M, Zinna G, Virgili A. Allergic contact dermatitis due to 1,3-dichloropropene soil fumigant. Contact Dermatitis 2003: 48: 341-342.

139. Sánchez-Pérez J, López M P, García-Díez A. Airborne allergic contact dermatitis from olaquindox in a rabbit breeder. Contact Dermatitis 2002: 46: 185.

140. Bonamonte D, Foti C, Cassano N, Rigano L, Angelini G. Contact dermatitis from organophosphorus pesticides. Contact Dermatitis 2001: 44: 179-180.
141. Nakamura M, Miyachi Y. Airborne photocontact dermatitis due to the insecticide phoxim. Contact Dermatitis 2003: 49: $105-106$

142. Uchino Y. A case of contact dermatitis due to feed for pig. Environ Dermatol (Japan) 2003: 10: 83.

143. Nishioka K, Takahata H. Contact allergy due to propineb. Contact Dermatitis 2000: 43: 310.

144. Kato A, Shoji A, Aoki N. Contact sensitivity to cigarettes. Contact Dermatitis 2005: 53: 52-53.

145. Anibarro P C, Brenosa B G, Madoz S E, Figueroa B E G, Muruzabal M T A, Bacalcoa M T L, Sanchez N L, Purroy A I T. Occupational airborne allergic contact dermatitis from disperse dyes. Contact Dermatitis 2000: 43: 44.

146. Marty C L, Cheng J F. Granuloma annulare presenting as contact dermatitis. Dermatitis 2005: 16: 34-37.

147. Saary M J, House R A, Holness D L. Dermatitis in a particleboard manufacturing facility. Contact Dermatitis 2001: 44: 325-330.

148. Lazarov A, Yair M, Lael E, Baitelman L. Airborne irritant contact dermatitis from phosphates in a fertilizer factory. Contact Dermatitis 2002: 46: 53-54.

149. Patiwael J A, Wintzen M, Rustemeyer T, Bruynzeel D P. Airborne irritant contact dermatitis due to synthetic fibres from an air-conditioning filter. Contact Dermatitis 2005: 52: 126-129.

150. Lensen G J, Jungbauer F H W, Goncalo M, Coenraads P J. Chlorothalonil: outbreak of airborne occupational irritant facial contact dermatitis. Contact Dermatitis 2006: 55: 17.

151. Walker S L, Ead R D, Beck M H. Occupational photoallergic contact dermatitis in a pharmaceutical worker manufacturing carprofen, a canine nonsteroidal antiinflammatory drug. Br J Dermatol 2005: 153: 58.

152. Lotti L, Difonzo E M, Francalanci S. Photoallergic contact dermatitis with chlorpromazine: report of two cases. Contact Dermatitis 2006: 55: 47.

153. Condé-Salazar L, Guimaraens D, González M A, Mancebo E. Occupational allergic contact urticaria from amoxicillin. Contact Dermatitis 2001: 45: 109.

154. Lidlle M, Hull C, Liu C, Powell D. Contact urticaria from curcumin. Dermatitis 2006: 17: 196-197.

155. Kanerva L, Pelttari M, Jolanki R, Alanko K, Estlander T, Suhonen R. Occupational contact urticaria from diglycidyl ether of bisphenol A epoxy resin. Allergy 2002: 57: 1205-1207.

156. Yung A, Papworth-Smith J, Wilkinson S M. Occupational contact urticaria from the solid-phase peptide synthesis coupling agents HATU and HBTU. Contact Dermatitis 2003: 49: 108-109.

157. Alanko K, Hannu T, Kuuliala O, Keskinen H. Allergic contact urticaria and anaphylaxis from occupational airborne exposure to HBTU. Exog Dermatol 2004: 3: 193.

158. Baur X, Gahnz G. Berufsbedingte aerogene Kontakurtikaria auf Hyazinthen. Dermatosen in Beruf und Umwelt 2002: 50: $238-240$.

159. Vega J M, Moneo I, Armentia A, Vega J, de la Fuente R, Fernández A. Pine processionary caterpillar as a new cause of immunologic contact urticaria. Contact Dermatitis 2000: 43: $129-132$.

160. Maier H, Spiegel W, Kinaciyan T, Krehan H, Cabaj A, Schopf A, Hönigsmann $\mathrm{H}$. The oak processionary caterpillar as the cause of an epidemic airborne disease: survey and analysis. Br J Dermatol 2003: 149: 990-997.

161. Fuentes Aparicio V, de Barrio Fernandez M, Rubio Sotes M, Rodriguez Paredes A, Martinez Molero M I, Zapatero Remon L, Bartolome Zavala B. Non-occupational allergy caused by the pine processionary caterpillar (Thaumetopoea pityocampa). Allergol Immunopathol (Madr) 2004: 32: 69-75.

162. Spiegel W, Maier H, Maier M. A non-infectious airborne disease. Lancet 2004: 363: 1438. 
163. Aparicio V F, Remon L Z, Molero M I, Lebreros E A, Mazuecos J M B, Zavala B B. Allergy to pine processionary caterpillar (Thaumetopoea pityocampa) in children. Allergol Immunopathol (Madr) 2006: 34: 59-63.

164. Gottschling S, Meyer S. An epidemic airborne disease caused by the oak processionary caterpillar. Pediatr Dermatol 2006: 23: 64-66.

165. Kanerva L, Estlander T, Petman L, Mäkinen-Kiljunen S. Occupational allergic contact urticaria to yucca (Yucca aloifolia), weeping fig (Ficus benjamina), and spathe flower (Spathiphyllum wall_isii). Allergy 2001: 56: 1008-1011.

166. Munno G, Giannoccaro F, Riva G, Antonicelli L. Allergy to yucca. Allergy 2001: 56: 921-922.

167. Scala E, Giani M, Pirrotta L, Guerra E C, Cadoni S, Girardelli C R, De Pità O, Puddu P. Occupational generalised urticaria and allergic airborne asthma due to anisakis simplex. Eur J Dermatol 2001: 11: 249-250.

168. Acciai M C, Macchia D, Sertoli A, Calabretta V M, Vanni E, Resti A, Carnevalli C. Occupational airborne sensitivity to Anisakis simplex in a fishmomger. Annali Italiani di Dermatologia alergologica 2005: 59: 118-120.

169. Uter W, Nohle M, Randerath B, Schwanitz H J. Occupational contact urticaria and late-phase bronchial asthma caused by compositae pollen in a florist. Am J Contact Dermat 2001: 12: 182-184.

170. Valks R, Conde-Salazar L, Barrantes O L. Occupational allergic contact urticaria and asthma from diphenylmethane4,4'-diisocyanatae Contact Dermatitis 2003: 49: 166-167.

171. Rodríguez A, De Barrio M, De Frutos C, de Benito V, Baeza M L. Occupational allergy to fern. Allergy 2001: 56: 89-90.

172. Krakowiak A, Palczynski C. Contact urticaria and respiratory symptoms induced by indirect contact with goat dander: a case report on vet's wife exposure. Contact Dermatitis 2005: 52: 168-169.
173. Kanerva L, Vanhanen M. Occupational allergic contact urticaria and rhino-conjunctivitis from a detergent protease. Contact Dermatitis 2001: 45: 49-51.

174. Parisot L, Aparicio C, Moneret-Vautrin D A, Guerin L. Allergy to lupine flour. Allergy 2001: 56: 918-919.

175. Torresani C, Zendri E, Vescovi V, De Panfilis G. Contact urticaria syndrome from occupational triphenyl phosphite exposure. Contact Dermatitis 2003: 48: 237-238.

176. Llombart B, Revert M A, Sastre A, Durá M, Pelaez A, Jordá E. Occupational protein contact dermatitis from flour and cuttlefish. Contact Dermatitis 2003: 49: 268-269.

177. Alvarez-Cuesta C, Ortiz G G, Díaz E R, Barrios S B, Osuna C G, Aguado C R, Pineda F. Occupational asthma and IgE-mediated contact dermatitis from sapele wood. Contact Dermatitis 2004: 51: 88-98.

178. Tjiu J-W, Chu C-Y, Sun C-C. 1,2-Ethanedithiol-induced erythema multiforme-like contact dermatitis. Acta Derm Venereol 2004: 84: 393-396.

179. Roest M A B, Powell S. Erythema multiforme-like eruption due to Japanese lacquer tree (Rhus verniciflua). Br J Dermatol 2003: 149: 102

180. Jovanović M, Mimica-Dukić N, Poljački M, Boža P. Erythema multiforme due to contact with weeds: a recurrence after patch testing. Contact Dermatitis 2003: 48: 17-25.

Address:

A. Goossens

Department of Dermatology

University Hospital

Catholic University Leuven

B-3000 Leuven

Belgium

Tel: 003216337860

Fax: 003216337012

e-mail:an.goossens@uz.kuleuven.ac.be 
This document is a scanned copy of a printed document. No warranty is given about the accuracy of the copy. Users should refer to the original published version of the material. 